\title{
Instantaneous phase synchronization of two decoupled quantum limit-cycle oscillators induced by conditional photon detection
}

\author{
Yuzuru Kato $\odot^{*}$ and Hiroya Nakao $\odot$ \\ Department of Systems and Control Engineering, Tokyo Institute of Technology, Tokyo 152-8552, Japan
}

(Received 17 September 2020; accepted 12 January 2021; published 28 January 2021)

\begin{abstract}
We show that conditional photon detection induces instantaneous phase synchronization between two decoupled quantum limit-cycle oscillators. We consider two quantum van der Pol oscillators without mutual coupling, each with additional single-photon dissipation, and perform continuous measurement of photon counting on the output fields of the two baths interacting through a beam splitter. It is observed that in-phase or antiphase coherence of the two decoupled oscillators instantaneously increases after photon detection and then decreases gradually in the weak quantum regime or quickly in the strong quantum regime until the next photon detection occurs. In the strong quantum regime, quantum entanglement also increases after photon detection and quickly disappears. We derive the analytical upper bounds for the increases in the quantum entanglement and phase coherence by conditional photon detection in the quantum limit.
\end{abstract}

DOI: 10.1103/PhysRevResearch.3.013085

\section{INTRODUCTION}

Synchronization phenomena, first reported by Huygens in the 17 th century, are widely observed in various areas of science and engineering, including laser oscillations, mechanical vibrations, oscillatory chemical reactions, and biological rhythms [1-6]. While synchronization of coupled or periodically driven nonlinear oscillators has been extensively investigated [1-3,7], decoupled oscillators that do not involve any interactions or periodic forcing can also exhibit synchronous behaviors when driven by common random forcing, such as the consistency or reproducibility of laser oscillations and spiking neocortical neurons receiving identical sequences of random signals [8,9]. Common-noise-induced synchronization has been theoretically investigated for decoupled limit-cycle oscillators subjected, e.g., to common random impulses [10-12] and Gaussian white noise [13-15].

Recent developments in nanotechnology have inspired theoretical investigations of quantum synchronization [16-41], and the first experimental demonstration of quantum phase synchronization in spin-1 atoms [42] and on the IBM Q system [43] has been reported very recently. Many studies have analyzed coupled quantum nonlinear dissipative oscillators, for example, synchronization of quantum van der Pol (vdP) oscillators [16-18], synchronization of ensembles of atoms [19], synchronization of triplet spins [20], measures for quantum synchronization of two oscillators [21-23], and synchronization blockade [24,25]. The effects of quantum measurement backaction on quantum nonlinear dissipative

\footnotetext{
${ }^{*}$ Corresponding author: kato.y.bg@m.titech.ac.jp

Published by the American Physical Society under the terms of the Creative Commons Attribution 4.0 International license. Further distribution of this work must maintain attribution to the author(s) and the published article's title, journal citation, and DOI.
}

oscillators have also been investigated as a unique feature of quantum systems, including improvement in the accuracy of Ramsey spectroscopy through the measurement of synchronized atoms [26], a measurement-induced transition between in-phase and antiphase synchronized states [27], unraveling of nonclassicality in optomechanical oscillators [44], characterization of synchronization using quantum trajectories [28], realization of quantum relaxation oscillators [29], and enhancement of synchronization by quantum measurement and feedback control [30].

In this paper, inspired by the common-noise-induced synchronization of decoupled classical oscillators, we consider the phase synchronization of two decoupled quantum oscillators induced by the common backaction of quantum measurement. We consider two quantum van der Pol oscillators without mutual coupling, each with additional single-photon dissipation, and perform a continuous measurement of photon counting on the output fields of the two baths interacting through a beam splitter. It is demonstrated that the quantum measurement backaction of conditional photon detection common to both oscillators induces the instantaneous phase synchronization of the oscillators.

\section{MODEL}

A schematic of the physical setup is depicted in Fig. 1. The stochastic master equation (SME) of the system can be expressed as

$$
\begin{aligned}
d \rho= & \mathcal{L}_{0} \rho d t+\mathcal{G}\left[L_{+}\right] \rho\left(d N_{+}-\gamma_{3} \operatorname{Tr}\left[L_{+}^{\dagger} L_{+} \rho\right] d t\right) \\
& +\mathcal{G}\left[L_{-}\right] \rho\left(d N_{-}-\gamma_{3} \operatorname{Tr}\left[L_{-}^{\dagger} L_{-} \rho\right] d t\right), \\
\mathcal{L}_{0} \rho= & \sum_{j=1,2}\left(-i\left[\omega a_{j}^{\dagger} a_{j}, \rho\right]+\gamma_{1} \mathcal{D}\left[a_{j}^{\dagger}\right] \rho\right. \\
& \left.+\gamma_{2} \mathcal{D}\left[a_{j}^{2}\right] \rho+\gamma_{3} \mathcal{D}\left[a_{j}\right] \rho\right)
\end{aligned}
$$




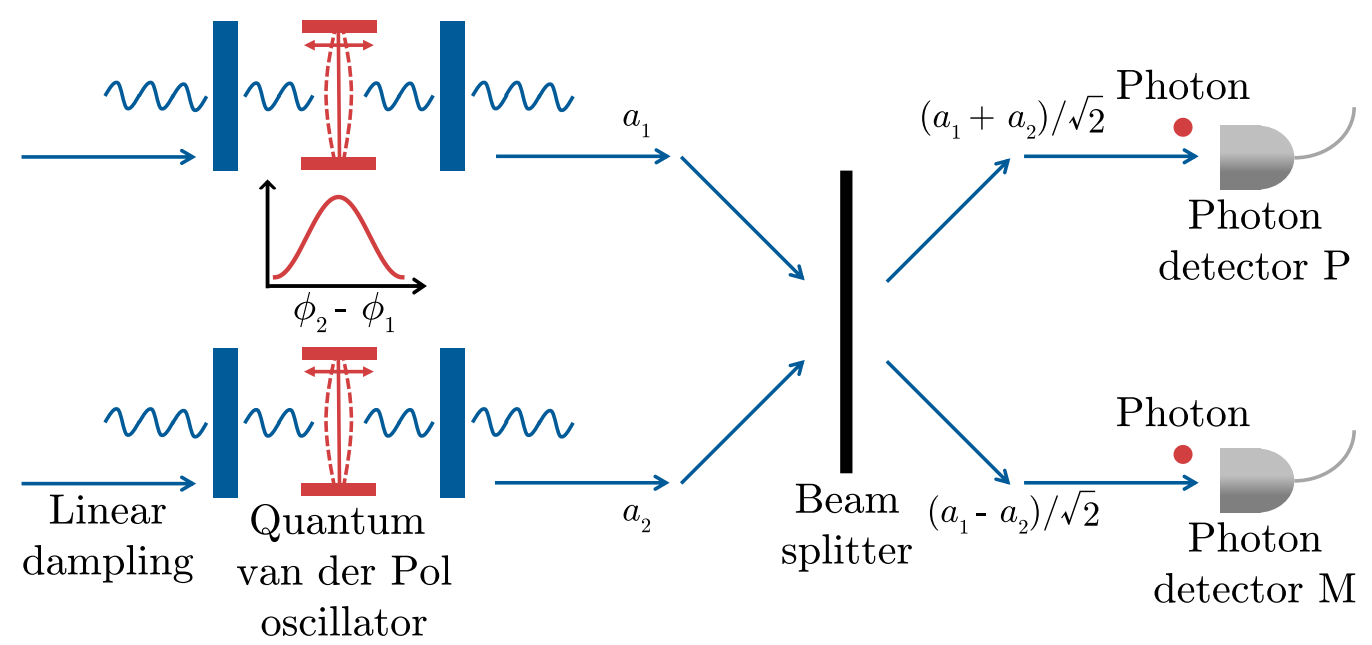

FIG. 1. Instantaneous phase synchronization of two decoupled quantum vdP oscillators induced by conditional photon detection. Either in-phase or antiphase coherence is induced after photon detection at detector $\mathrm{P}$ or $\mathrm{M}$, respectively.

$$
\begin{gathered}
L_{ \pm}=\frac{1}{\sqrt{2}}\left(a_{1} \pm a_{2}\right), \quad \mathcal{D}[L] \rho=L \rho L^{\dagger}-\frac{1}{2}\left(\rho L^{\dagger} L+L^{\dagger} L \rho\right), \\
\mathcal{G}[L] \rho=\frac{L \rho L^{\dagger}}{\operatorname{Tr}\left[L \rho L^{\dagger}\right]}-\rho,
\end{gathered}
$$

where the natural frequency $\omega$ and the decay rates $\gamma_{1}, \gamma_{2}$, and $\gamma_{3}$ for negative damping, nonlinear damping, and linear damping, respectively, are assumed identical for both oscillators, $N_{ \pm}$are two independent Poisson processes whose increments are given by $d N_{ \pm}=1$ with probability $\gamma_{3} \operatorname{Tr}\left[L_{ \pm}^{\dagger} L_{ \pm} \rho\right] d t$ and $d N_{ \pm}=0$ with probability $1-\gamma_{3} \operatorname{Tr}\left[L_{ \pm}^{\dagger} L_{ \pm} \rho\right] d t$ in each interval $d t$, where $d N_{+}=1$ and $d N_{-}=1$ represent the photon detection at detectors $\mathrm{P}$ and $\mathrm{M}$ in Fig. 1, respectively, and the reduced Planck constant is set to $\hbar=1$.

In the derivation of the SME (1), the SLH framework [45,46], a general formulation for quantum networked systems, has been used to describe the cascade and concatenate connections of quantum system components. In this framework, the quantum system is specified by parameters $(\boldsymbol{S}, \boldsymbol{L}, H)$, i.e., a scattering matrix $\boldsymbol{S}$, coupling vector $\boldsymbol{L}$, and Hamiltonian $H$, from which the SME (1) can be derived by using the quantum filtering theory $[47,48]$. See the Appendix for details of the SLH framework and the derivation of the SME (1).

Note that if we average Eq. (1) over various stochastic trajectories, we obtain two independent master equations without measurement for completely decoupled oscillators whose phase values are fully incoherent in the steady state. Conditional photon detection at the detector $\mathrm{P}$ and $\mathrm{M}$ by the operators $L_{+}=\left(a_{1}+a_{2}\right) / \sqrt{2}$ and $L_{-}=\left(a_{1}-a_{2}\right) / \sqrt{2}$ after the beam splitter can also be interpreted as an unraveling of two competing dissipative coupling terms $\mathcal{D}\left[a_{1}+a_{2}\right]$ and $\mathcal{D}\left[a_{1}-a_{2}\right]$ considered in Refs. [17,49], which induce synchronization of the two oscillators.

\section{WEAK QUANTUM REGIME}

First, we numerically analyze the quantum SME (1) in the weak quantum regime. To characterize the degree of phase coherence between two quantum vdP oscillators, we use the absolute value of the normalized correlator [27]

$$
S_{12}=\left|S_{12}\right| e^{i \theta_{12}}=\frac{\operatorname{Tr}\left[a_{1}^{\dagger} a_{2} \rho\right]}{\sqrt{\operatorname{Tr}\left[a_{1}^{\dagger} a_{1} \rho\right] \operatorname{Tr}\left[a_{2}^{\dagger} a_{2} \rho\right]}}
$$

as the order parameter, which is a quantum analog of the order parameter for two classical noisy oscillators [3]. The modulus $\left|S_{12}\right|$ takes values in $0 \leqslant\left|S_{12}\right| \leqslant 1 ;\left|S_{12}\right|=1$ when the two oscillators are perfectly phase synchronized and $\left|S_{12}\right|=0$ when they are perfectly phase incoherent. We also use the argument $\theta_{12}$ to characterize the averaged phase difference of the two oscillators in order to distinguish in-phase and antiphase coherence. We use the negativity $\mathcal{N}=\left(\left\|\rho^{\Gamma_{1}}\right\|_{1}-1\right) / 2$ to quantify the quantum entanglement of the two oscillators, where $\rho^{\Gamma_{1}}$ represents the partial transpose of the system with respect to the subsystem representing the first oscillator and $\|X\|_{1}=\operatorname{Tr}|X|=\operatorname{Tr} \sqrt{X^{\dagger} X}$ [50,51]. When $\mathcal{N}$ takes a nonzero value, the two oscillators are entangled with one other. We also observe the purity $P=\operatorname{Tr}\left[\rho^{2}\right]$.

Figures 2(a)-2(d) plot the time evolution of $\left|S_{12}\right|, \theta_{12}, \mathcal{N}$, and $P$ in the weak quantum regime, respectively, calculated for a single trajectory of the quantum SME (1). As shown in Fig. 2(a), $\left|S_{12}\right|$ instantaneously increases after detection of a photon either at $\mathrm{P}$ or $\mathrm{M}$, indicating that the phase coherence of the two decoupled oscillators is induced by conditional photon detection. After photon detection, $\left|S_{12}\right|$ gradually decreases because the two oscillators converge to the desynchronized steady state of the SME (1) in the absence of photon detection, i.e., $d N_{ \pm}=0$.

In this regime, the nonlinear damping is not strong and the relaxation to the desynchronized state is relatively slow. Therefore, subsequent photon detection typically occurs before the convergence to the desynchronized state and $\left|S_{12}\right|$ remains always positive. Figure 2(b) shows that $\theta_{12}$ takes either $\theta_{12}=0$ or $\theta_{12}=\pi$. This indicates that the two oscillators immediately attain in-phase coherence after photon detection at $\mathrm{P}$ or antiphase coherence after photon detection at $\mathrm{M}$. The negativity and purity are shown in Figs. 3(c) and 3(d), respectively, where the negativity is always zero and the purity takes 

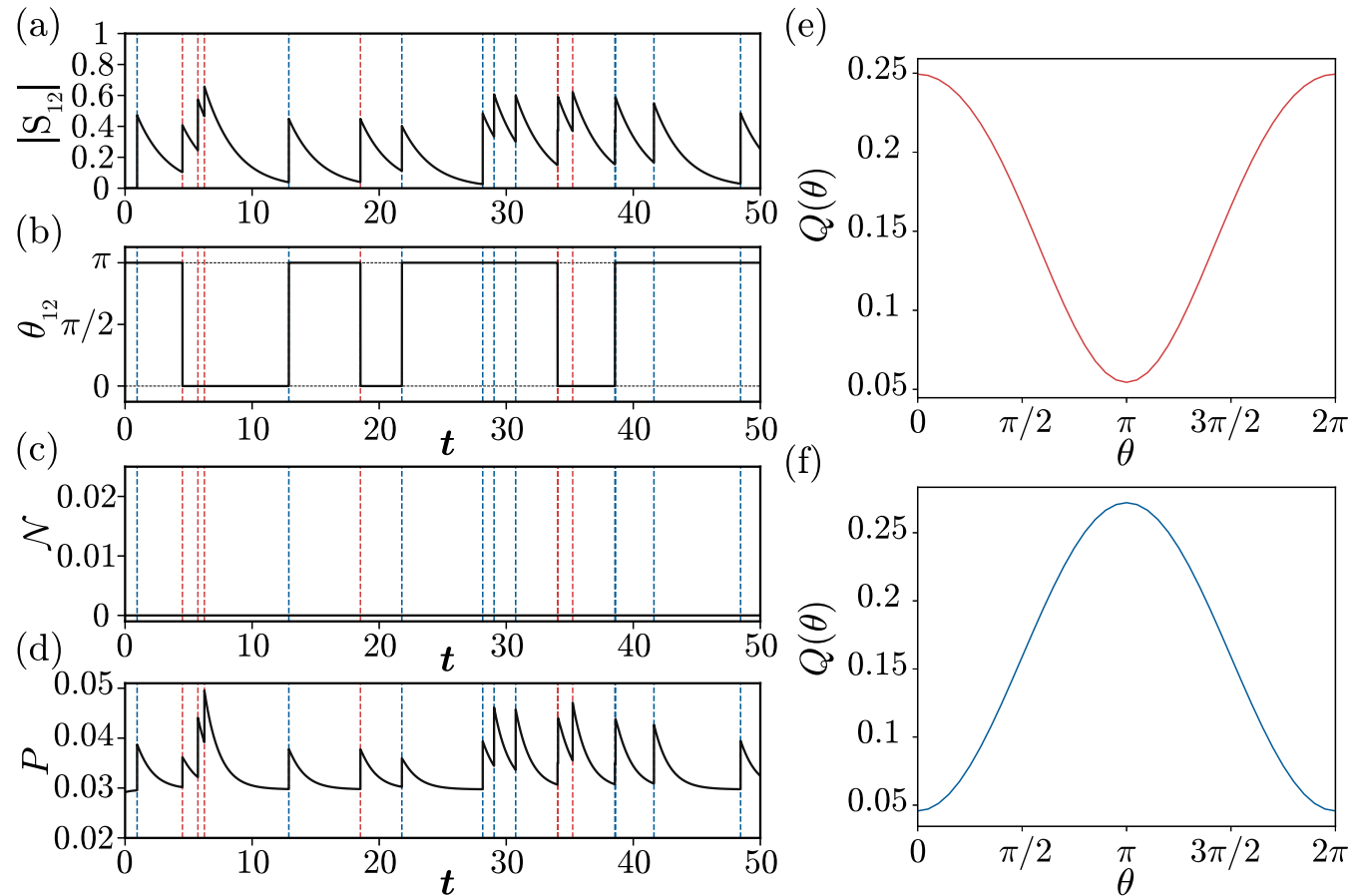

FIG. 2. Results in the weak quantum regime. The parameters are $\left(\omega, \gamma_{2}, \gamma_{3}\right) / \gamma_{1}=(0.1,0.25,0.1)$ with $\gamma_{1}=1$. (a)-(d) Time evolution of (a) the absolute value of the normalized correlator $\left|S_{12}\right|$, (b) the average phase value $\theta_{12}$, (c) negativity $\mathcal{N}$, and (d) purity $P$. (e), (f) $Q$ distributions $Q(\theta)$ immediately after the first photon detection at (e) $\mathrm{P}(t=4.51)$ and (f) $\mathrm{M}(t=0.95)$. Photon detection at detectors $\mathrm{P}$ and $\mathrm{M}$ is indicated by the red- and blue-dashed lines in (a)-(d), respectively.
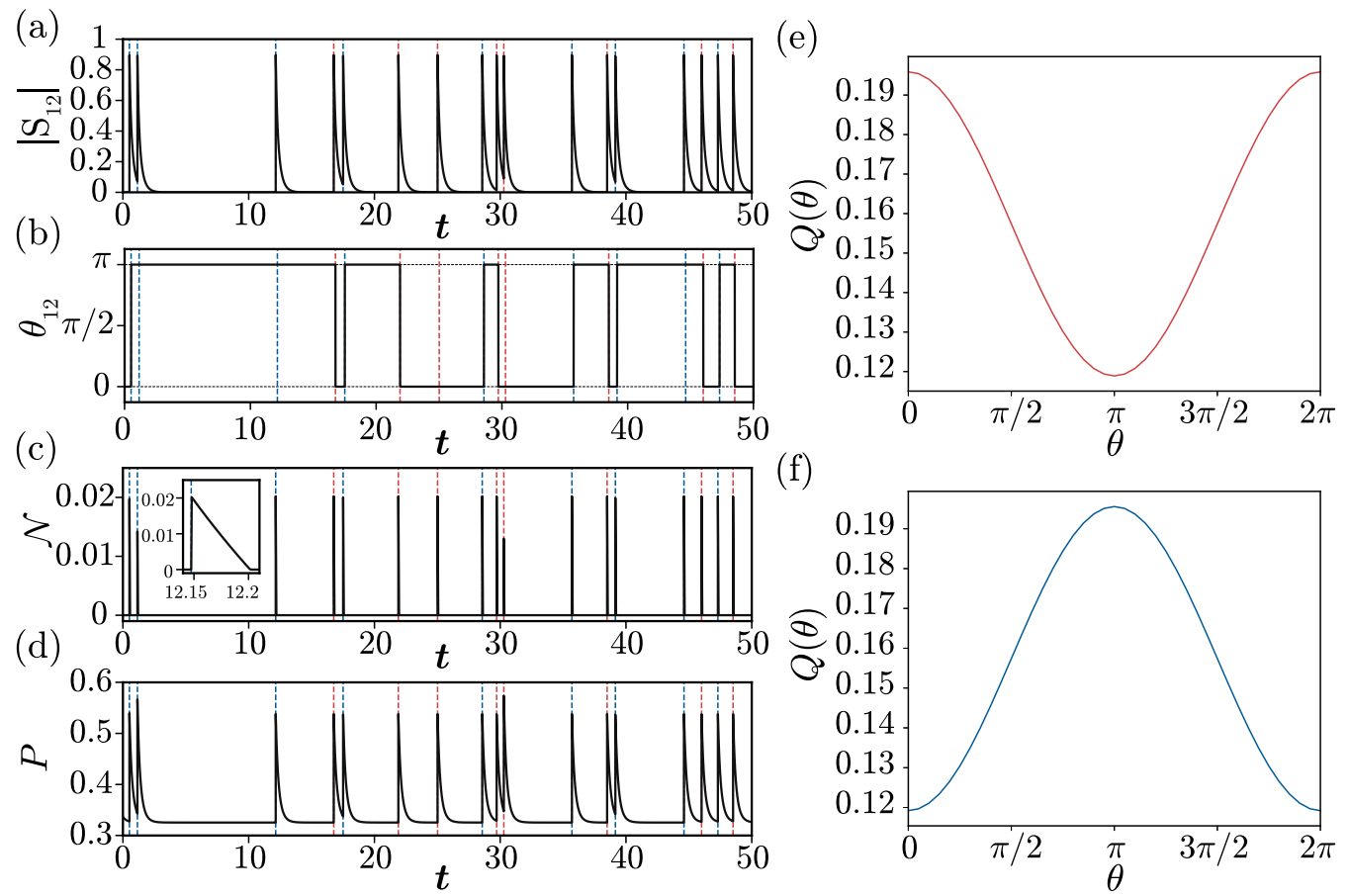

FIG. 3. Results in the strong quantum regime. The parameters are $\left(\omega, \gamma_{2}, \gamma_{3}\right) / \gamma_{1}=(0.5,50,0.5)$ with $\gamma_{1}=1$. (a)-(d) Time evolution of (a) the absolute value of the normalized correlator $\left|S_{12}\right|$, (b) the averaged phase value $\theta_{12}$, (c) negativity $\mathcal{N}$, and (d) purity $P$. (e), (f) $Q$ distributions $Q(\theta)$ immediately after the first photon detection at (e) $\mathrm{P}(t=16.8)$ and (f) $\mathrm{M}(t=0.52)$. Photon detection at detectors $\mathrm{P}$ and $\mathrm{M}$ is indicated by the red- and blue-dashed lines in (a)-(d), respectively. 
small values between 0.03 and 0.05 , indicating that the system is separable and mixed.

The phase coherence of the two oscillators can also be captured by using the Hushimi $Q$ distribution of the phase difference $\theta=\phi_{2}-\phi_{1}$ [52] between the two oscillators, $Q(\theta)$, calculated by introducing the two-mode $Q$ distribution [53] $Q\left(\alpha_{1}, \alpha_{1}^{*}, \alpha_{2}, \alpha_{2}^{*}\right)=\frac{1}{\pi^{2}}\left\langle\alpha_{1}, \alpha_{2}|\rho| \alpha_{1}, \alpha_{2}\right\rangle$ with $R_{j} e^{i \phi_{j}}=$ $\alpha_{j}(j=1,2)$ and integrating over $R_{1}, R_{2}$, and $\phi_{1}+\phi_{2}$.

Figures 2(e) and 2(f) show $Q(\theta)$ of the system states immediately after the first photon detection at detectors $\mathrm{P}$ and $\mathrm{M}$, respectively. The peak of $Q(\theta)$ occurs at $\theta=0$ in Fig. 2(e) and at $\theta=\pi$ in Fig. 2(f), clearly indicating that the in-phase and antiphase coherences of the two oscillators are induced by conditional photon detection.

\section{STRONG QUANTUM REGIME}

We next analyze the quantum SME (1) in a stronger quantum regime. Figures 3(a)-3(d) show the evolution of $\left|S_{12}\right|, \theta_{12}$, $\mathcal{N}$, and $P$, respectively. As shown in Fig. 3(a), $\left|S_{12}\right|$ takes large values close to 1 immediately after photon detection, indicating that instantaneous phase coherence also arises in this case. In this regime, the nonlinear damping is strong and the system quickly converges to the desynchronized steady state of the SME (1) when detection does not occur, i.e., $d N_{ \pm}=0$. Therefore, phase coherence quickly disappears and $\left|S_{12}\right|$ remains zero until the next photon detection occurs.

Similar to Fig. 2(b), Fig. 3(b) shows that $\theta_{12}$ takes either $\theta_{12}=0$ or $\theta_{12}=\pi$. Thus, the two oscillators become in-phase coherent after photon detection at $\mathrm{P}$ and antiphase coherent after photon detection at M. Remarkably, Figs. 3(c) and 3(d) show that nonzero negativity and purity with values between 0.5 and 0.6 are attained instantaneously after photon detection, indicating that mixed entangled states are obtained in this case. However, the quantum entanglement quickly disappears as shown in the inset in Fig. 3(c). Here, the apparent linear decay of the negativity is due to the large decay rate and the cutoff at zero of the negativity (see, e.g., Ref. [54] for a discussion about the decay of the negativity). Figures 3(e) and 3(f) show the $Q$ distributions $Q(\theta)$ of the system states immediately after the first photon detection at detectors $\mathrm{P}$ and $\mathrm{M}$, respectively. The $Q$ distributions are peaked at $\theta=0$ and $\theta=\pi$, clearly indicating that in-phase and antiphase coherence of the two oscillators are induced also in this case.

\section{QUANTUM LIMIT}

From previous numerical results, it is expected that maximum quantum entanglement is attained in the quantum limit, i.e., $\gamma_{2} \rightarrow \infty$. In this limit, we can map the quantum vdP oscillator to an analytically tractable two-level system with basis states $|0\rangle$ and $|1\rangle$ [17], and transform the SME (1) to

$$
\begin{aligned}
d \rho= & \mathcal{L}_{0}^{q} \rho d t+\mathcal{G}\left[L_{+}^{q}\right] \rho\left(d N_{+}-\gamma_{3} \operatorname{Tr}\left[L_{+}^{q^{\dagger}} L_{+}^{q} \rho\right] d t\right) \\
& +\mathcal{G}\left[L_{-}^{q}\right] \rho\left(d N_{-}-\gamma_{3} \operatorname{Tr}\left[L_{-}^{q^{\dagger}} L_{-}^{q} \rho\right] d t\right) \\
\mathcal{L}_{0}^{q} \rho= & \sum_{j=1,2}\left(-i\left[\omega \sigma_{j}^{+} \sigma_{j}^{-}, \rho\right]+\gamma_{1} \mathcal{D}\left[\sigma_{j}^{+}\right] \rho\right. \\
& \left.+\left(2 \gamma_{1}+\gamma_{3}\right) \mathcal{D}\left[\sigma_{j}^{-}\right] \rho\right) \\
L_{ \pm}^{q}= & \frac{1}{\sqrt{2}}\left(\sigma_{1}^{-} \pm \sigma_{2}^{-}\right)
\end{aligned}
$$

with $\sigma_{j}^{-}=|0\rangle\left\langle\left. 1\right|_{j}\right.$ and $\left.\sigma_{j}^{+}=\mid 1\right\rangle\left\langle\left. 0\right|_{j}\right.$ representing the lowering and raising operators of the $j$ th $\operatorname{system}(j=1,2)$, respectively, because the transition $|1\rangle \stackrel{2 \gamma_{1}}{\longrightarrow}|2\rangle \stackrel{2 \gamma_{2}}{\longrightarrow}|0\rangle$ can be regarded as $|1\rangle \stackrel{2 \gamma_{1}}{\longrightarrow}|0\rangle$ when $\gamma_{2} \rightarrow \infty$.

The steady state of Eq. (3) without detection, i.e., $d N_{ \pm}=$ 0 , can be analytically obtained, which is given by a diagonal matrix $\rho^{\text {pre }}=\operatorname{diag}\left(\rho_{0}^{\text {pre }}, \rho_{1}^{\text {pre }}, \rho_{1}^{\text {pre }}, \rho_{2}^{\text {pre }}\right)$ with

$$
\begin{aligned}
& \rho_{0}^{\text {pre }}=\frac{(k-3) \sqrt{k^{2}+2 k+9}+k^{2}-2 k+9}{2 k^{2}}, \\
& \rho_{1}^{\text {pre }}=\frac{3 \sqrt{k^{2}+2 k+9}-k-9}{2 k^{2}}, \\
& \rho_{2}^{\text {pre }}=\frac{-(k+3) \sqrt{k^{2}+2 k+9}+k^{2}+4 k+9}{2 k^{2}} .
\end{aligned}
$$

Note that only a single parameter $k=\gamma_{3} / \gamma_{1}$ specifies the elements of the matrix, where we assume $k>0$, namely, photon detection occurs with a nonzero probability.

The states $\rho_{ \pm}^{\text {pos }}=L_{ \pm}^{q} \rho^{\text {pre }} L_{ \pm}^{q_{\dagger}^{\dagger}} / \operatorname{Tr}\left[L_{ \pm}^{q} \rho^{\text {pre }} L_{ \pm}^{q^{\dagger}}\right]$, immediately after photon detection occurs at detectors $\mathrm{P}\left(\rho_{+}^{\text {pos }}\right)$ and $\mathbf{M}$ $\left(\rho_{-}^{\text {pos }}\right)$, can be represented by a density matrix,

$$
\rho_{ \pm}^{\text {pos }}=\rho_{0}^{\text {pos }}|00\rangle\langle 00|+\rho_{1}^{\text {pos }}\left(\frac{|01\rangle \pm|10\rangle}{\sqrt{2}}\right)\left(\frac{\langle 01| \pm\langle 10|}{\sqrt{2}}\right),
$$

with

$$
\begin{aligned}
\rho_{0}^{\mathrm{pos}} & =\frac{-3 \sqrt{k^{2}+2 k+9}+k+9}{k\left(\sqrt{k^{2}+2 k+9}-k-3\right)}, \\
\rho_{1}^{\mathrm{pos}} & =\frac{(k+3) \sqrt{k^{2}+2 k+9}-k^{2}-4 k-9}{k\left(\sqrt{k^{2}+2 k+9}-k-3\right)} .
\end{aligned}
$$

Using this result, we can explicitly calculate the normalized correlator $S_{12}$ and the $Q$ distribution of the phase difference between the two oscillators. If subsequent photon detection does not occur, the state after photon detection in Eq. (6) converges to the steady state $\rho_{\text {pre }}$ in Eq. (4) with the approximate decay rate determined by $\gamma_{1}$ and $\gamma_{3}$ in Eq. (3). In this case, the correlator $S_{12}$ of the states $\rho_{ \pm}^{\text {pos }}$ immediately after photon detection always takes $S_{12}= \pm 1$ irrespective of the value of $k$ (and then quickly decays).

The $Q$ distribution for $\rho_{ \pm}^{\text {pos }}$ can also be calculated as (a similar calculation for the Wigner distribution of the phase difference has been performed in Ref. [17])

$$
Q(\theta)\left[\rho_{ \pm}^{\mathrm{pos}}\right]=\frac{1}{2 \pi} \pm \frac{\rho_{1}^{\mathrm{pos}} \cos \theta}{8} .
$$

These results qualitatively agree with the corresponding results in the strong quantum regime in Fig. 3. It is notable that the dependence of the phase coherence on $k$ can be captured by the peak height of $Q(\theta)$ but not by the normalized correlator $S_{12}$ in the quantum limit. Indeed, the element $\rho_{0}^{\text {pos }}|00\rangle\langle 00|$ in Eq. (5) affects $Q(\theta)$ (through $\rho_{1}^{\text {pos }}=1-\rho_{0}^{\text {pos }}$ ) in Eq. (7), whereas it does not affect the value of $S_{12}$.

The above result indicates that the degree of phase coherence is better quantified by the peak height of $Q(\theta)$ rather than $S_{12}$ in strong quantum regimes. This is because $S_{12}$ is defined as a quantum analog of the order parameter for the coherence 

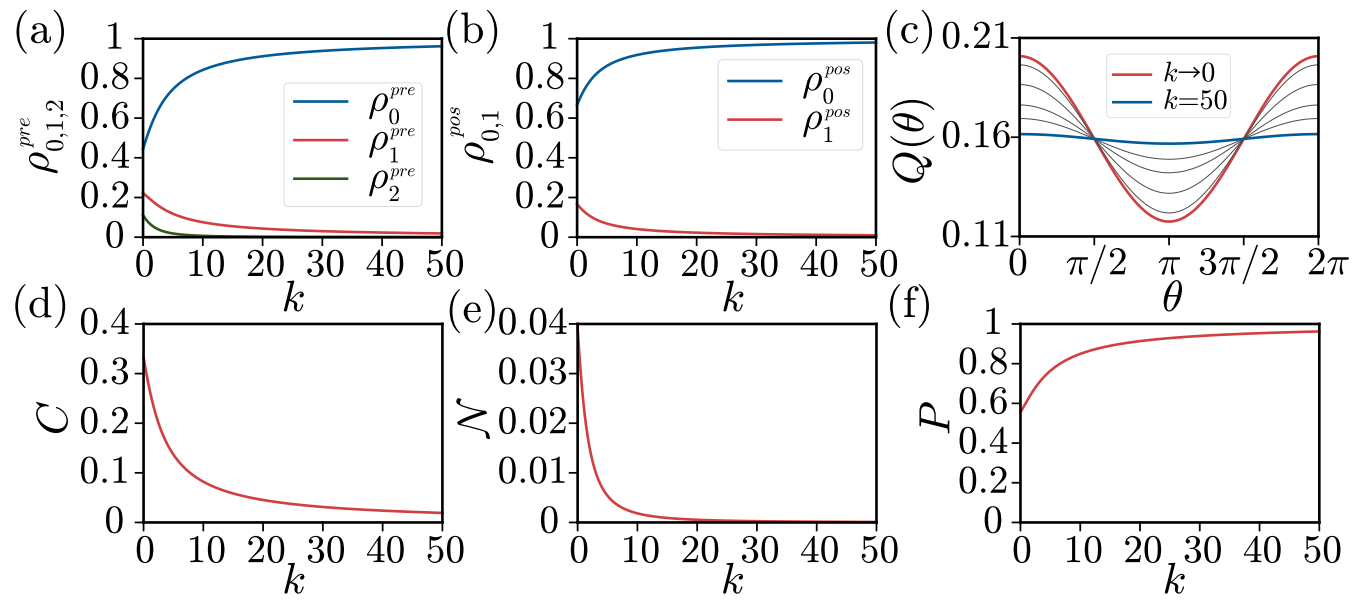

FIG. 4. Dependence of the results on the parameter $k=\gamma_{3} / \gamma_{1}$ of the two-level system in the quantum limit $\gamma_{2} \rightarrow \infty$. (a) Elements of $\rho^{\text {pre }}$. (b) Elements of $\rho^{\text {pos }}$. (c) $Q(\theta)\left[\rho_{+}^{\text {pos }}\right]$ distributions of the phase difference of two oscillators for $k \rightarrow 0$ (red line), $k=0.5,2,5,10$ (gray lines from the red line to the blue line), and $k=50$ (blue line) are shown. (d) Concurrence. (e) Negativity. (f) Purity.

of classical noisy oscillators, which is quantitatively correct only in the semiclassical regime. This observation is also important in interpreting the results in the weak and strong quantum regimes shown in Figs. 2 and 3, where $Q(\theta)$ in the weak quantum regime (Fig. 2) are more sharply peaked than those in the strong quantum regime (Fig. 3), while $\left|S_{12}\right|$ in Fig. 2 takes smaller values than that in Fig. 3. Thus, $S_{12}$ may not work well for comparing the phase coherence between different quantum regimes.

In the quantum limit, the symmetric superpositions $|S\rangle=$ $(|01\rangle+|10\rangle) / \sqrt{2}$ and $|A\rangle=(|01\rangle-|10\rangle) / \sqrt{2}$ can be regarded as in-phase and antiphase synchronized states, because the corresponding distributions $Q(\theta)[|S\rangle\langle S|]=\frac{1}{2 \pi}+\frac{\cos \theta}{8}$ and $Q(\theta)[|A\rangle\langle A|]=\frac{1}{2 \pi}-\frac{\cos \theta}{8}$ are peaked at $\theta=0$ and $\theta=\pi$, respectively. As $|A\rangle$ and $|S\rangle$ are dark states with respect to $L_{+}^{q}$ and $L_{-}^{q}$, i.e., $L_{+}^{q}|A\rangle=0$ and $L_{-}^{q}|S\rangle=0$, photon detection at detector $\mathrm{P}$ annihilates the antiphase-synchronized state $|A\rangle$ and creates an in-phase-synchronized state $|S\rangle$ with $S_{12}=1$ $\left(\theta_{12}=0\right)$, while photon detection at detector $\mathrm{M}$ annihilates $|S\rangle$ and creates $|A\rangle$ with $S_{12}=-1\left(\theta_{12}=\pi\right)$.

Figures 4(a)-4(c) show the dependence of the elements $\rho^{\text {pre }}$ and $\rho^{\text {pos }}$ and $Q(\theta)\left[\rho_{+}^{\text {pos }}\right]$ on $k$, respectively [we only plot $Q(\theta)\left[\rho_{+}^{\mathrm{pos}}\right]$ because $\left.Q(\theta)\left[\rho_{-}^{\mathrm{pos}}\right]=Q(\theta+\pi)\left[\rho_{+}^{\mathrm{pos}}\right]\right]$. As shown in Fig. 4(a), $\rho_{1}^{\text {pre }}$ and $\rho_{2}^{\text {pre }}$ take larger values when $k$ is smaller.

When $k \rightarrow 0, \rho_{1}^{\text {pre }}$ and $\rho_{2}^{\text {pre }}$ approach the supremum values, $\rho_{1}^{\text {pre }} \rightarrow \frac{2}{9}$ and $\rho_{2}^{\text {pre }} \rightarrow \frac{1}{9}\left(\rho_{0}^{\text {pre }} \rightarrow \frac{4}{9}\right)$, corresponding to the completely incoherent steady state of the two decoupled quantum vdP oscillators in the quantum limit, i.e., $\rho^{\text {pre }} \rightarrow$ $\left(\frac{2}{3}|0\rangle\left\langle 0\left|+\frac{1}{3}\right| 1\right\rangle\langle 1|\right) \otimes\left(\frac{2}{3}|0\rangle\left\langle 0\left|+\frac{1}{3}\right| 1\right\rangle\langle 1|\right)$, where $Q(\theta)$ is uniform $[16,17]$. Therefore, $\rho_{1}^{\text {pos }}$ approaches the supremum value, $\rho_{1}^{\text {pos }} \rightarrow \frac{1}{3}\left(\rho_{0}^{\text {pos }} \rightarrow \frac{2}{3}\right)$, as shown in Fig. 4(b), and $Q(\theta)\left[\rho_{+}^{\text {pos }}\right]$ exhibits the maximum peak as shown in Fig. 4(c), indicating that maximum phase coherence is obtained. In the opposite limit, $k \rightarrow \infty$, $\rho^{\text {pre }}$ converges to the two-mode vacuum state $\rho^{\text {pre }} \rightarrow|00\rangle\langle 00|$, i.e., $\rho_{1}^{\text {pre }}, \rho_{2}^{\text {pre }} \rightarrow 0\left(\rho_{0}^{\text {pre }} \rightarrow 1\right)$, resulting in $\rho_{1}^{\text {pos }} \rightarrow 0\left(\rho_{0}^{\text {pos }} \rightarrow 1\right)$ and the uniform distribution $Q(\theta)\left[\rho_{+}^{\mathrm{pos}}\right] \rightarrow \frac{\pi}{2}$. Note that we can only consider the limit $k \rightarrow 0$ (no photon detection occurs when $k=0$ ), and that the two-mode vacuum state in the $k \rightarrow \infty$ limit is not a limit cycle.
In addition to the negativity $\mathcal{N}$ and purity $P$, the quantum entanglement of the density matrix $\rho_{\text {pos }}$ in Eq. (5) can also be quantified using the concurrence [55] $C=$ $\max \left(0, \lambda_{1}-\lambda_{2}-\lambda_{3}-\lambda_{4}\right)$, where $\lambda_{1}, \lambda_{2}, \lambda_{3}$, and $\lambda_{4}$ are the square roots of the eigenvalues of $\rho \tilde{\rho}$ with $\tilde{\rho}=$ $\left(\sigma_{y} \otimes \sigma_{y}\right) \rho^{*}\left(\sigma_{y} \otimes \sigma_{y}\right)$ in decreasing order. The concurrence $C$ takes a nonzero value when the two oscillators are entangled with each other $(C \in[0,1]$ by definition).

Figures 4(d)-4(f) show the dependence of $C, \mathcal{N}$, and $P$ on $k$ for $\rho_{ \pm}^{\text {pos }}$, respectively. Note that $C, \mathcal{N}$, and $P$ take the same values for both $\rho_{+}$and $\rho_{-}$. In the limit $k \rightarrow 0, C, \mathcal{N}$, and $P$ approach the upper bounds as $C \rightarrow \frac{1}{3}, \mathcal{N} \rightarrow \frac{\sqrt{5}-2}{6}$, and $P \rightarrow$ $\frac{5}{9}$. In the opposite limit $k \rightarrow \infty$, these values converge as $C \rightarrow$ $0, \mathcal{N} \rightarrow 0$, and $P \rightarrow 1$, which corresponds to those quantities for the two-mode vacuum states.

Photon detection occurs less frequently when $k$ is smaller, because the probability of photon detection in the interval $d t$ at detectors $\mathrm{P}$ or $\mathrm{M}$ is given by $k \gamma_{1} \operatorname{Tr}\left[L_{ \pm}^{\dagger} L_{ \pm} \rho\right] d t$. Therefore, on average, an infinitely long observation time is required before photon detection to approach the upper bounds for the degree of phase coherence and quantum entanglement in the limit $k \rightarrow 0$.

\section{CONCLUDING REMARKS}

We have analyzed two decoupled quantum van der Pol oscillators and demonstrated that quantum measurement backaction of conditional photon detection induces instantaneous phase synchronization of the oscillators. In-phase or antiphase coherence between the oscillators has been observed instantaneously after photon detection, which decays gradually in the weak quantum regime or quickly in the strong quantum regime until the next photon detection. In the strong quantum regime, a short-time increase in the quantum entanglement has also been observed. In the quantum limit, we analytically obtained the upper bounds for the increase in quantum entanglement and phase coherence.

In this paper, we presented only the results for the case with two identical oscillators under the measurement 
without inefficiency. For the case with oscillators whose natural frequencies are slightly different, we also confirmed numerically that almost the same results as in Figs. 2 and 3 are obtained except for the phase difference shown in Fig. 2(b) or 3 (b); in this case, the phase difference between the oscillators increases or decreases with a constant rate (i.e., with the frequency difference) between the jumps to 0 or $\pi$ caused by photon detection. The results in the quantum limit analyzed in Sec. V are also independent of the natural frequencies of the two oscillators. As for the measurement inefficiency [56], we confirmed numerically that it mainly affects the frequency of photon detection. This is because the probability of photon detection is proportional to the measurement efficiency, whereas the system state just after photon detection is not strongly affected as the $d N_{ \pm}$terms in Eq. (1) are independent of the measurement efficiency.

Recently, physical implementations of the quantum vdP oscillator with ion trap systems $[16,17]$ and optomechanical systems $[18,32]$ have been discussed. Additional single-photon dissipation and photon detectors can also be introduced $[53,57]$. The physical setup considered in the present study does not require explicit mutual coupling between the oscillators. Therefore, it can, in principle, be implemented by using existing experimental methods and provide a method for generating the phase-coherent states of quantum limit-cycle oscillators.

\section{ACKNOWLEDGMENTS}

The numerical simulations were performed by using the QUTIP numerical toolbox [58]. We acknowledge JSPS KAKENHI Grants No. JP17H03279, No. JP18H03287, No. JPJSBP120202201, No. JP20J13778, and JST CREST Grant No. JP-MJCR1913 for financial support.

\section{APPENDIX: SLH FRAMEWORK}

In this Appendix, we derive the SME (1) using the SLH framework to describe cascade and concatenate connections of the quantum system components $[45,46]$. In this framework, the parameters in the time evolution of a quantum system $\rho$ are specified by $\boldsymbol{G}=(\boldsymbol{S}, \boldsymbol{L}, H)$ with

$$
\boldsymbol{S}=\left(\begin{array}{ccc}
S_{11} & \cdots & S_{1 n} \\
\vdots & \vdots & \vdots \\
S_{n 1} & \cdots & S_{n n}
\end{array}\right), \quad \boldsymbol{L}=\left(\begin{array}{c}
L_{1} \\
\vdots \\
L_{n}
\end{array}\right)
$$

where $\boldsymbol{S}$ is the scattering matrix with operator entries satisfying $\boldsymbol{S}^{\dagger} \boldsymbol{S}=\boldsymbol{S} \boldsymbol{S}^{\dagger}=\boldsymbol{I}^{n}, \boldsymbol{L}$ is a coupling vector with operator entries, and $H$ is a self-adjoint operator referred to as the system Hamiltonian. We denote by $\boldsymbol{I}^{n}$ an identity matrix with $n$ dimensions.

With these parameters, the time evolution of the system obeys the master equation

$$
\frac{d \rho}{d t}=-i[H, \rho]+\sum_{i=1}^{n} \mathcal{D}\left[L_{i}\right] \rho,
$$

where $S$ is involved in the calculation of the cascade and concatenation products and has an important role in determining the forms of $H$ and $\boldsymbol{L}$ of the whole network system consisting (a)

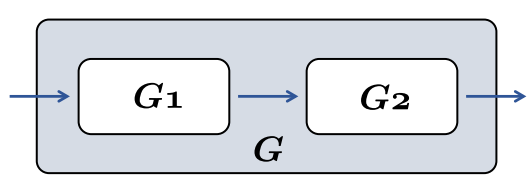

Cascade

Connection

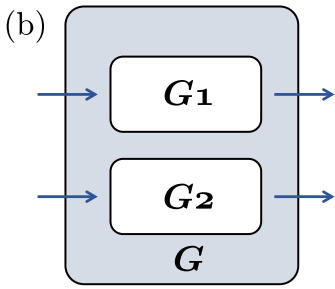

Concatenate

Connection
FIG. 5. (a) Cascade and (b) concatenate connections of the two system components $G_{1}$ and $G_{2}$.

of the system components. This specification of parameters is based on Hudson-Parthasarathy's work [59].

The cascade product [Fig. 5(a)] of $\boldsymbol{G}_{1}=\left(\boldsymbol{S}_{1}, \boldsymbol{L}_{1}, H_{1}\right)$ and $\boldsymbol{G}_{2}=\left(\boldsymbol{S}_{2}, \boldsymbol{L}_{1}, H_{2}\right)$ is given by

$$
\begin{aligned}
\boldsymbol{G}_{1} \triangleleft \boldsymbol{G}_{2}= & \left(\boldsymbol{S}_{2} \boldsymbol{S}_{1}, \boldsymbol{L}_{2}+\boldsymbol{S}_{2} \boldsymbol{L}_{1}, H_{1}+H_{2}\right. \\
& \left.+\frac{1}{2 i}\left(\boldsymbol{L}_{2}^{\dagger} \boldsymbol{S}_{2} \boldsymbol{L}_{1}-\boldsymbol{L}_{1}^{\dagger} \boldsymbol{S}_{2}^{\dagger} \boldsymbol{L}_{2}\right)\right),
\end{aligned}
$$

and the concatenation product [see Fig. 5(b)] of $\boldsymbol{G}_{1}$ and $\boldsymbol{G}_{2}$ is given by

$$
\boldsymbol{G}_{1} \boxplus \boldsymbol{G}_{2}=\left[\left(\begin{array}{cc}
\boldsymbol{S}_{1} & 0 \\
0 & \boldsymbol{S}_{2}
\end{array}\right),\left(\begin{array}{l}
\boldsymbol{L}_{1} \\
\boldsymbol{L}_{2}
\end{array}\right), H_{1}+H_{2}\right] .
$$

Our aim is to derive the SME (1) of the physical setup depicted in Fig. $1[45,46]$. To this end, we denote $\boldsymbol{G}_{j}^{Q V D P}$ as the parameters of the $j$ th quantum vdP oscillator with an additional single-photon dissipation,

$$
\boldsymbol{G}_{j}^{Q V D P}=\left[\boldsymbol{I}^{3},\left(\begin{array}{c}
\sqrt{\gamma_{1}} a_{j}^{\dagger} \\
\sqrt{\gamma_{2}} a_{j}^{2} \\
\sqrt{\gamma_{3}} a_{j}
\end{array}\right), \omega a_{j}^{\dagger} a_{j}\right] .
$$

The concatenate connection of $\boldsymbol{G}_{1}^{Q V D P}$ and $\boldsymbol{G}_{2}^{Q V D P}$ is

$$
\boldsymbol{G}_{1}^{Q V D P} \boxplus \boldsymbol{G}_{2}^{Q V D P}=\left[\boldsymbol{I}^{6},\left(\begin{array}{c}
\sqrt{\gamma_{1}} a_{1}^{\dagger} \\
\sqrt{\gamma_{1}} a_{2}^{\dagger} \\
\sqrt{\gamma_{2}} a_{1}^{2} \\
\sqrt{\gamma_{2}} a_{2}^{2} \\
\sqrt{\gamma_{3}} a_{1} \\
\sqrt{\gamma_{3}} a_{2}
\end{array}\right), \sum_{j=1,2} \omega a_{j}^{\dagger} a_{j}\right],
$$

where we have changed the order of the elements in $\boldsymbol{L}$ for simplicity of notation.

In this study, we consider a 50:50 beam splitter. The parameters of the beam splitter $\boldsymbol{G}^{\mathrm{BS}}$ for the output fields of the two baths

$$
\left.\boldsymbol{G}^{\mathrm{BS}}=\left[\begin{array}{ll}
\boldsymbol{I}^{4} & \boldsymbol{O}^{42} \\
\boldsymbol{O}^{24} & \left(\begin{array}{cc}
\frac{1}{\sqrt{2}} & -\frac{1}{\sqrt{2}} \\
\frac{1}{\sqrt{2}} & \frac{1}{\sqrt{2}}
\end{array}\right)
\end{array}\right), 0,0\right],
$$

where we denote by $\boldsymbol{O}^{n m}$ a zero matrix with the dimensions $n \times m$. 
The cascading connection of the two above-mentioned components is given by

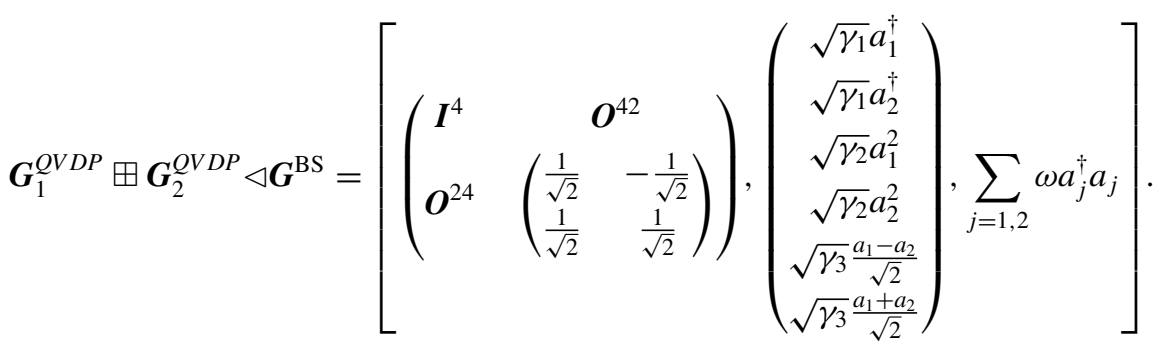

Using transformation $\mathcal{D}\left[\frac{a_{1}+a_{2}}{\sqrt{2}}\right] \rho+\mathcal{D}\left[\frac{a_{1}-a_{2}}{\sqrt{2}}\right] \rho=\mathcal{D}\left[a_{1}\right] \rho+\mathcal{D}\left[a_{2}\right] \rho$, the quantum master equation (A2) with the parameters given in Eq. (A8) gives $d \rho=\mathcal{L}_{0} \rho d t$ of the SME (1). Then, using the quantum filtering theory [47,48], SME (1) can be obtained.

[1] A. T. Winfree, The Geometry of Biological Time (Springer, New York, 2001).

[2] Y. Kuramoto, Chemical Oscillations, Waves, and Turbulence (Springer, Berlin, 1984).

[3] A. Pikovsky, M. Rosenblum, and J. Kurths, Synchronization: A Universal Concept in Nonlinear Sciences (Cambridge University Press, Cambridge, UK, 2001).

[4] H. Nakao, Phase reduction approach to synchronisation of nonlinear oscillators, Contemp. Phys. 57, 188 (2016).

[5] G. B. Ermentrout and D. H. Terman, Mathematical Foundations of Neuroscience (Springer, New York, 2010).

[6] S. Strogatz, Nonlinear Dynamics and Chaos (Westview Press, Boulder, CO, 1994).

[7] D. G. Aronson, G. B. Ermentrout, and N. Kopell, Amplitude response of coupled oscillators, Physica D 41, 403 (1990).

[8] A. Uchida, R. McAllister, and R. Roy, Consistency of Nonlinear System Response to Complex Drive Signals, Phys. Rev. Lett. 93, 244102 (2004).

[9] Z. F. Mainen and T. J. Sejnowski, Reliability of spike timing in neocortical neurons, Science 268, 1503 (1995).

[10] A. S. Pikovskii, Synchronization and stochastization of array of self-excited oscillators by external noise, Radiophys. Quantum Electron. 27, 390 (1984).

[11] H. Nakao, K.-s. Arai, K. Nagai, Y. Tsubo, and Y. Kuramoto, Synchrony of limit-cycle oscillators induced by random external impulses, Phys. Rev. E 72, 026220 (2005).

[12] K. Arai and H. Nakao, Phase coherence in an ensemble of uncoupled limit-cycle oscillators receiving common poisson impulses, Phys. Rev. E 77, 036218 (2008).

[13] J.-n. Teramae and D. Tanaka, Robustness of the Noise-Induced Phase Synchronization in a General Class of Limit Cycle Oscillators, Phys. Rev. Lett. 93, 204103 (2004).

[14] D. S. Goldobin and A. S. Pikovsky, Synchronization of selfsustained oscillators by common white noise, Physica A 351, $126(2005)$

[15] H. Nakao, K. Arai, and Y. Kawamura, Noise-Induced Synchronization and Clustering in Ensembles of Uncoupled Limit-Cycle Oscillators, Phys. Rev. Lett. 98, 184101 (2007).

[16] T. E. Lee and H. Sadeghpour, Quantum Synchronization of Quantum van der Pol Oscillators with Trapped Ions, Phys. Rev. Lett. 111, 234101 (2013).
[17] T. E. Lee, C.-K. Chan, and S. Wang, Entanglement tongue and quantum synchronization of disordered oscillators, Phys. Rev. E 89, 022913 (2014).

[18] S. Walter, A. Nunnenkamp, and C. Bruder, Quantum synchronization of two van der Pol oscillators, Ann. Phys. 527, 131 (2015).

[19] M. Xu, D. A. Tieri, E. Fine, J. K. Thompson, and M. J. Holland, Synchronization of Two Ensembles of Atoms, Phys. Rev. Lett. 113, 154101 (2014).

[20] A. Roulet and C. Bruder, Quantum Synchronization and Entanglement Generation, Phys. Rev. Lett. 121, 063601 (2018).

[21] A. Mari, A. Farace, N. Didier, V. Giovannetti, and R. Fazio, Measures of Quantum Synchronization in Continuous Variable Systems, Phys. Rev. Lett. 111, 103605 (2013).

[22] V. Ameri, M. Eghbali-Arani, A. Mari, A. Farace, F Kheirandish, V. Giovannetti, and R. Fazio, Mutual information as an order parameter for quantum synchronization, Phys. Rev. A 91, 012301 (2015).

[23] F. Galve, G. L. Giorgi, and R. Zambrini, Quantum correlations and synchronization measures, in Lectures on General Quantum Correlations and their Applications (Springer, Berlin, 2017) pp. 393-420.

[24] N. Lörch, S. E. Nigg, A. Nunnenkamp, R. P. Tiwari, and C. Bruder, Quantum Synchronization Blockade: Energy Quantization Hinders Synchronization of Identical Oscillators, Phys. Rev. Lett. 118, 243602 (2017).

[25] S. E. Nigg, Observing quantum synchronization blockade in circuit quantum electrodynamics, Phys. Rev. A 97, 013811 (2018).

[26] M. Xu and M. Holland, Conditional Ramsey Spectroscopy with Synchronized Atoms, Phys. Rev. Lett. 114, 103601 (2015).

[27] T. Weiss, A. Kronwald, and F. Marquardt, Noise-induced transitions in optomechanical synchronization, New J. Phys. 18, 013043 (2016).

[28] N. Es'haqi Sani, G. Manzano, R. Zambrini, and R. Fazio, Synchronization along quantum trajectories, Phys. Rev. Research 2, 023101 (2020).

[29] A. Chia, L. Kwek, and C. Noh, Relaxation oscillations and frequency entrainment in quantum mechanics, Phys. Rev. E 102, 042213 (2020).

[30] Y. Kato and H. Nakao, Enhancement of quantum synchronization via continuous measurement and feedback control, New J. Phys. 23, 013007 (2021). 
[31] M. Koppenhöfer and A. Roulet, Optimal synchronization deep in the quantum regime: Resource and fundamental limit, Phys. Rev. A 99, 043804 (2019).

[32] S. Walter, A. Nunnenkamp, and C. Bruder, Quantum Synchronization of a Driven Self-Sustained Oscillator, Phys. Rev. Lett. 112, 094102 (2014).

[33] S. Sonar, M. Hajdušek, M. Mukherjee, R. Fazio, V. Vedral, S. Vinjanampathy, and L.-C. Kwek, Squeezing Enhances Quantum Synchronization, Phys. Rev. Lett. 120, 163601 (2018).

[34] N. Lörch, E. Amitai, A. Nunnenkamp, and C. Bruder, Genuine Quantum Signatures in Synchronization of Anharmonic SelfOscillators, Phys. Rev. Lett. 117, 073601 (2016).

[35] T. Weiss, S. Walter, and F. Marquardt, Quantum-coherent phase oscillations in synchronization, Phys. Rev. A 95, 041802(R) (2017).

[36] A. Roulet and C. Bruder, Synchronizing the Smallest Possible System, Phys. Rev. Lett. 121, 053601 (2018).

[37] D. Witthaut, S. Wimberger, R. Burioni, and M. Timme, Classical synchronization indicates persistent entanglement in isolated quantum systems, Nat. Commun. 8, 14829 (2017).

[38] Y. Kato, N. Yamamoto, and H. Nakao, Semiclassical phase reduction theory for quantum synchronization, Phys. Rev. Research 1, 033012 (2019).

[39] Y. Kato and H. Nakao, Semiclassical optimization of entrainment stability and phase coherence in weakly forced quantum limit-cycle oscillators, Phys. Rev. E 101, 012210 (2020).

[40] Y. Kato and H. Nakao, Quantum asymptotic phase reveals signatures of quantum synchronization, arXiv:2006.00760.

[41] W.-K. Mok, L.-C. Kwek, and H. Heimonen, Synchronization boost with single-photon dissipation in the deep quantum regime, Phys. Rev. Research 2, 033422 (2020).

[42] A. W. Laskar, P. Adhikary, S. Mondal, P. Katiyar, S. Vinjanampathy, and S. Ghosh, Observation of Quantum Phase Synchronization in Spin-1 Atoms, Phys. Rev. Lett. 125, 013601 (2020).

[43] M. Koppenhöfer, C. Bruder, and A. Roulet, Quantum synchronization on the IBM Q system, Phys. Rev. Research 2, 023026 (2020).

[44] M. Koppenhöfer, C. Bruder, and N. Lörch, Unraveling nonclassicality in the optomechanical instability, Phys. Rev. A 97, 063812 (2018).
[45] J. Gough and M. R. James, The series product and its application to quantum feedforward and feedback networks, IEEE Trans. Autom. Control 54, 2530 (2009).

[46] J. Combes, J. Kerckhoff, and M. Sarovar, The SLH framework for modeling quantum input-output networks, Adv. Phys.: X 2, 784 (2017).

[47] R. Van Handel, J. K. Stockton, and H. Mabuchi, Feedback control of quantum state reduction, IEEE Trans. Autom. Control 50, 768 (2005).

[48] L. Bouten, R. Van Handel, and M. R. James, An introduction to quantum filtering, SIAM J. Control Optim. 46, 2199 (2007).

[49] K. Ishibashi and R. Kanamoto, Oscillation collapse in coupled quantum van der Pol oscillators, Phys. Rev. E 96, 052210 (2017).

[50] K. Życzkowski, P. Horodecki, A. Sanpera, and M. Lewenstein, Volume of the set of separable states, Phys. Rev. A 58, 883 (1998).

[51] G. Vidal and R. F. Werner, Computable measure of entanglement, Phys. Rev. A 65, 032314 (2002).

[52] K. Husimi, Some formal properties of the density matrix, Proc. Phys.-Math. Soc. Jpn. 22, 264 (1940).

[53] H. J. Carmichael, Statistical Methods in Quantum Optics 1, 2 (Springer, New York, 2007).

[54] J.-L. Guo, H. Li, and G.-L. Long, Decoherent dynamics of quantum correlations in qubit-qutrit systems, Quantum Inf. Process. 12, 3421 (2013).

[55] W. K. Wootters, Entanglement of Formation of an Arbitrary State of Two Qubits, Phys. Rev. Lett. 80, 2245 (1998).

[56] K. Jacobs and D. A. Steck, A straightforward introduction to continuous quantum measurement, Contemp. Phys. 47, 279 (2006).

[57] H. M. Wiseman and G. J. Milburn, Quantum Measurement and Control (Cambridge University Press, Cambridge, UK, 2009).

[58] J. Johansson, P. Nation, and F. Nori, QuTiP: An open-source PYTHON framework for the dynamics of open quantum systems, Comput. Phys. Commun. 183, 1760 (2012); QuTiP 2: A PYTHON framework for the dynamics of open quantum systems, 184, 1234 (2013).

[59] R. L. Hudson and K. R. Parthasarathy, Quantum Ito's formula and stochastic evolutions, Commun. Math. Phys. 93, 301 (1984). 